Supplementary Information for

\title{
How Blinking Affects Photon Correlations in
}

\section{Multichromophoric Nanoparticles}

Tim Schröder, ${ }^{1}$ Sebastian Bange, ${ }^{2}$ Jakob Schedlbauer, ${ }^{2}$ Florian Steiner, ${ }^{1}$ John M. Lupton, ${ }^{2}$

Philip Tinnefeld, ${ }^{1}$ and Jan Vogelsang, ${ }^{2}$ *

${ }^{1}$ Department Chemie and Center for NanoScience (CeNS), Ludwig-Maximilians-Universität

München, Butenandtstr. 5-13, 81377 München, Germany

2Institut für Experimentelle und Angewandte Physik, Universität Regensburg,

Universitätsstrasse 31, 93040 Regensburg, Germany

KEYWORDS: single-molecule spectroscopy, photon statistics, DNA origami, photophysics, quantum optics 


\section{Details of DNA origami structures and sample preparation}

The DNA origami structure was modified using caDNAno (version 0.2.2, design schematics in Fig. S1). The scaffold is an 8064-nucleotide-long ssDNA extracted from M13mp18 bacteriophages. All staple strands as well as the dye labeled oligonucleotides were purchased from Eurofins Genomics GmbH (see the end of Supplementary Information). The ATTO 542-modified oligonucleotides for external labeling were purchased from biomers.net. Scaffold and oligonucleotides were mixed according to table S1 for origami folding. The folding buffer (FB) is a Tris-EDTA buffer $(1 \times$ TE, $10 \mathrm{mM}$ Tris-HCl, $1 \mathrm{mM}$ EDTA $\cdot \mathrm{Na}_{2}$ ) with $20 \mathrm{mM} \mathrm{MgCl}$ and $5 \mathrm{mM} \mathrm{NaCl}$. In the annealing process, the mixture was heated and slowly cooled down with a nonlinear thermal ramp over 16 hours according Nickels et al. ${ }^{1}$ After annealing, the excess staples were removed with polyethylene glycol (PEG) precipitation. The samples were mixed with an equal volume of PEG precipitation buffer (1× TAE, $15 \%(\mathrm{w} / \mathrm{v})$ PEG-8000, $500 \mathrm{mM}$ $\mathrm{NaCl}, 12 \mathrm{mM} \mathrm{MgCl}_{2}$ ) and centrifuged at $16 \mathrm{krcf}$ (thousand relative centrifugal force, i.e. $1000 \mathrm{~g}$ ) for 30 min at $4{ }^{\circ} \mathrm{C}$. After removing the supernatant, the pellet was suspended in $1 \times \mathrm{FB}$. Afterwards, the DNA origami was externally labeled with ATTO 542-modified oligonucleotides. A threefold excess with respect to the extended staples was used and the structure was incubated for $20 \mathrm{~min}$ in a wet chamber at room temperature. The DNA origami structures were purified via gel electrophoresis. A $1.5 \%$ agarose gel containing a Tris base, acetic acid and EDTA buffer $(0.5 \times$ TAE, $20 \mathrm{mM}$ Tris-HCl, $10 \mathrm{mM}$ acetic acid, $0.5 \mathrm{mM}$ EDTA) and $12 \mathrm{mM} \mathrm{MgCl} 2$ was used at $60 \mathrm{~V}$ for 2 hours in a gel box cooled in an icewater bath. The gel was not stained to avoid staining reagent-dye interactions. On a blue-illuminated table DNA origami structures could be seen due to the numerous ATTO 542 dyes. DNA origami structures were recovered from the target band. The samples were stored at $-26{ }^{\circ} \mathrm{C}$ until further use.

\section{Folding Table}

Final concentrations for DNA origami folding are given in Table S1. The meaning of the reagents is described below:

Table S1: Folding reagents with final concentrations.

\begin{tabular}{|l|l|}
\hline Reagent & Final concentration / nM \\
\hline scaffold & 25 \\
\hline core staples & 225 \\
\hline biotin staples & 250 \\
\hline extended staples & 225 \\
\hline dye and refill staples & 225 \\
\hline
\end{tabular}

scaffold: Single-stranded viral $8064 \mathrm{nt}$ ssDNA from M13mp18.

core staples: Contains every unmodified staples of the rectangular DNA origami. The wildtype structure is given in Ref. ${ }^{2}$.

biotin staples: Four biotin modified staples. Modifications are placed at the 3' end.

extended staples: 13 staples extended at the 3 ' end for external labeling. The extended sequence is: 5' TTTTCCTCTACCACCTACATCAC 3'. Sequence for the ATTO 542 oligonucleotides: 5' GTGATGTAGGTGGTAGAGGA-ATtO 542 3'

dye and refill staples: Oligonucleotides labeled with ATTO $647 \mathrm{~N}$ or ATTO 700 at the 5' end. 


\section{DNA origami structures}

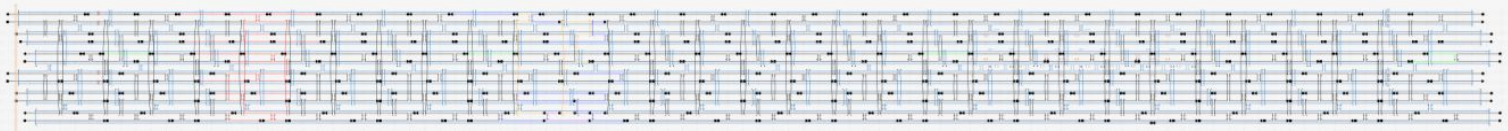

Figure S1. caDNAno sketch of the DNA origami structure used. Modified staples are colored. Green staples are biotin labeled. Red staples are extended at the 3' (5' TTTTCCTCTACCACCTACATCAC) end for external labeling with ATTO 542 modified oligonucleotides. Orange staples are labeled at the 5' end with ATTO $647 \mathrm{~N}$ violet ones with ATTO 700. Blue staples are next to the ATTO $647 \mathrm{~N}$ labeled staples to stabilize the structure.

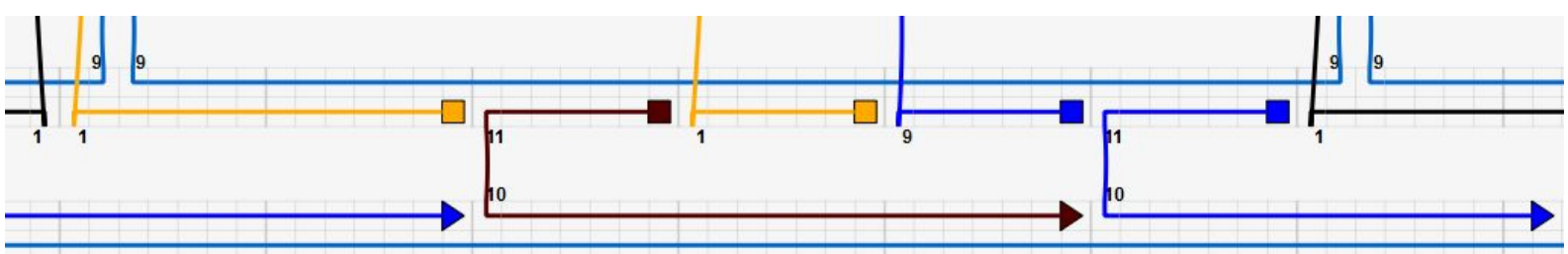

Figure S2. Close up of the DNA origami structure with two ATTO 647N (5' end of the orange staples) dyes separated by $6 \mathrm{~nm}$ and an ATTO 700 dye (5' end of the brown staple) for the collective blinking experiment.

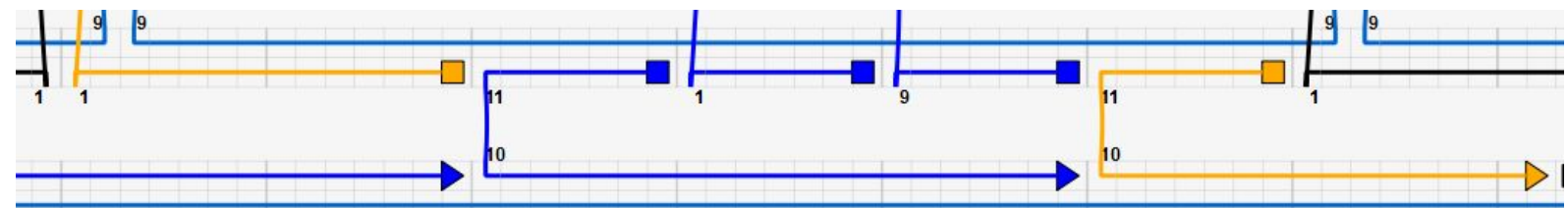

Figure S3. Close up of the DNA origami structure with two ATTO 647N (5' end of the orange staples) dyes separated by $12 \mathrm{~nm}$ for the independent blinking experiment.

\section{Simulations for Figures 1 and 4}

Simulations were performed with a self-written python script. The probability of detecting a photon was set to be 0.08 for intensity state $A$ and 0.00 for intensity state $B$. The photons were randomly distributed on two detection channels $C h 0$ and $C h 1$. The laser repetition rate was set to be $50 \mathrm{MHz}$ and $10^{7}$ laser pulses were simulated. The simulations were analyzed based on a correlation algorithm proposed by Laurence $e t$ al. $^{3}$ Positive lag times correspond to the cross correlation of $C h 0 \rightarrow C h 1$ and negative lag times correspond to the correlation of $\operatorname{Ch} 1 \rightarrow \operatorname{Ch} 0$.

For blinking dyes the switching rate between state $\mathrm{A}$ and $\mathrm{B}$ was fixed to $k_{\mathrm{A}}=k_{\mathrm{B}}=2 \cdot 10^{6} \mathrm{~s}^{-1}$ for each dye.

For Figure 4 the intensity levels were fixed to $I_{\mathrm{A}}=0.08$ and $I_{\mathrm{B}}=0.0016$. The fluorescence lifetime of the bright state was set to $\tau_{\mathrm{A}}=4 \mathrm{~ns}$ and the fluorescence lifetime of the dark state to $\tau_{\mathrm{B}}=0.8 \mathrm{~ns}$.

\section{Altering the correlation amplitude by excitation power for independently and collectively blinking dyes}


Figure S4a shows an exemplary time trace of two independently blinking ATTO 647N dyes separated by $12 \mathrm{~nm}$. With an oxygen scavenger and $2 \mathrm{mM}$ Trolox as reducing agent the dyes are pushed constantly into the radical anion state. The blue and red parts of the figure correspond to an excitation power of $4.3 \mathrm{~kW} / \mathrm{cm}^{2}$ and $1.1 \mathrm{~kW} / \mathrm{cm}^{2}$, respectively. We started with the high excitation intensity. After 11 seconds, we switched to lower excitation intensity and measured until one dye bleaches at $48 \mathrm{~s}$. We recorded much longer intensity time traces with lower excitation power to ensure an equal signal-tonoise ratio in the correlation calculation. The signal-to-noise ratio scales with the square of the fluorescence intensity.

Figure S4b and S4c show a two-second snapshot of the time trace in panel a, but with $1 \mathrm{~ms}$ binning. The intensity histogram in Figure S4b shows that most of the time, both dyes are in the non-fluorescent state for $4.3 \mathrm{~kW} / \mathrm{cm}^{2}$ excitation power. Figure $\mathrm{S} 4 \mathrm{c}$ shows that at lower excitation power, the cases for one or two dyes being in their fluorescent state are more prominent.

The cross correlations for the high and low excitation power are shown in S4d with a blue and a red curve, respectively. Higher excitation powers result in higher photon bunching amplitudes for independent dyes because the bunching amplitude scales linearly with the excitation intensity. As expected for two independently blinking dyes, however, $g^{(2)}(0)$ stays constant at $\sim 0.5$ for both excitation powers. For independently blinking dyes, the ratio $N_{\mathrm{c}} / N_{\ell}$ thus constitutes a pAB normalization that is inappropriate for chromophore counting.

a
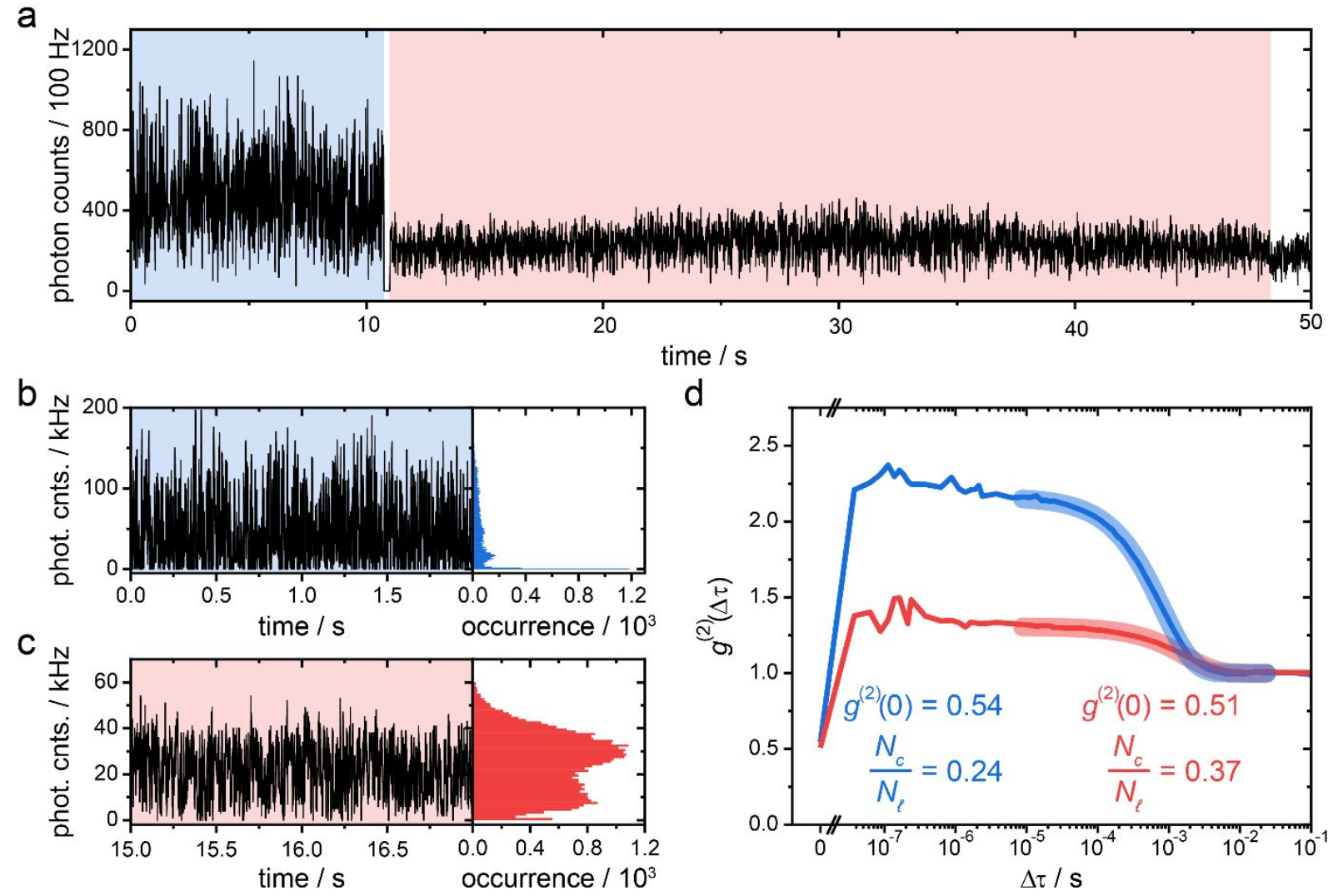

Figure S4. (a) Fluorescence time trace of two independently blinking ATTO $647 \mathrm{~N}$ dyes separated by $12 \mathrm{~nm}$. The excitation power was altered between $4.1 \mathrm{~kW} / \mathrm{cm}^{2}$ and $1.1 \mathrm{~kW} / \mathrm{cm}^{2}$ as indicated by blue and red background colors, respectively. (b) A two-second snippet of the fluorescence trajectory at 4.1 $\mathrm{kW} / \mathrm{cm}^{2}$ excitation with the corresponding intensity histogram. (c) A two-second snippet of the fluorescence trajectory at $1.1 \mathrm{~kW} / \mathrm{cm}^{2}$ excitation with corresponding intensity histogram. (d) Cross correlation of the fluorescence time trace excited with $4.1 \mathrm{~kW} / \mathrm{cm}^{2}$ and $1.1 \mathrm{~kW} / \mathrm{cm}^{2}$ shown in blue and red, respectively. Corresponding monoexponential fit from equation 9 are depicted in bold lines. 
To prove that the transition rate into the singlet manifold $k_{o n}$ is power independent, we extract the transition rates from the intensity correlations shown in Figure S4d. For the extraction of the rate constants we have to consider that the observed $g^{(2)}(\Delta \tau)$ correlation is a sum of two intensity correlations each representing one independently blinking dye molecule. Since both dyes experience a similar nano-environment and excitation power, the transition rates into the dark state, $k_{\text {off }}$, and back again, $k_{o n}$, are expected to be the same for both dyes, which is supported by the monoexponential decays seen in Figure S4d. If multiple independent intensity fluctuations are involved, each correlation is weighted with the square of the fractional intensity $\mathfrak{I}$ for each dye.

$$
\mathfrak{J}_{1}=\frac{I_{1}}{\sum I_{\mathrm{i}}} \text { eq. } 1
$$

Here, $\mathfrak{J}_{1}$ denotes the fractional intensity of the first dye with the measured intensity $I_{1}$. The total intensity correlation, $g_{\mathrm{total}}^{(2)}(\Delta \tau)$, can then be written as:

$$
g_{\text {total }}^{(2)}(\Delta \tau)=\sum \Im_{\mathrm{i}}^{2} g_{\mathrm{i}}^{(2)}(\Delta \tau)+1 \quad \text { eq. } 2
$$

In our case, the switching kinetics and intensities are the same for two independently blinking ATTO $647 \mathrm{~N}$ dyes. This results in a fractional intensity of $\mathfrak{J}_{1}=\mathfrak{J}_{2}=\frac{1}{2}$.

$$
g_{\text {total }}^{(2)}(\Delta \tau)=\left(\frac{1}{2}\right)^{2} g_{1}^{(2)}(\Delta \tau)+\left(\frac{1}{2}\right)^{2} g_{2}^{(2)}(\Delta \tau)+1 \quad \text { eq. } 3
$$

Both dyes have the same blinking kinetics and therefore show the same intensity correlation $g_{1}^{(2)}(\Delta \tau)=$ $g_{2}^{(2)}(\Delta \tau)$. Stochastic switching between an emitting and a non-emitting state results in a monoexponential decay in the intensity correlation.

$$
g_{\text {total }}^{(2)}(\Delta \tau)=\sum \Im_{\mathrm{i}}^{2}\left[C_{\mathrm{i}} \cdot \exp \left(-k_{\text {kineticsi }} \cdot \Delta \tau\right)\right]+1 \quad \text { eq. } 4
$$

For on-off-switching, the bunching amplitude $C$ equals the equilibrium constant

$$
C=K=\frac{k_{\text {off }}}{k_{\text {on }}} \quad \text { eq. } 5
$$

and the correlation relaxation rate $k_{\text {kinetics }}$ is the sum of the switching rates

$$
k_{\text {kinetics }}=k_{\text {on }}+k_{\text {off }} \quad \text { eq. } 6
$$

The transition rate constants are supposed to be the same for both dyes. Therefore, they will show the same bunching amplitude $C_{1}=C_{2}$ and the same correlation relaxation rate $k_{\text {kinetics } 1}=k_{\text {kinetics2 }}$. However, since both amplitudes are weighted by the square of the fractional intensity we have to consider this damping.

$$
\begin{gathered}
g_{\text {total }}^{(2)}(\Delta \tau)=1+\left(\frac{1}{2}\right)^{2}\left[C_{1} \cdot \exp \left(-k_{\text {kinetics } 1} \cdot \Delta \tau\right)\right]+\left(\frac{1}{2}\right)^{2}\left[C_{2} \cdot \exp \left(-k_{\text {kinetics } 2} \cdot \Delta \tau\right)\right] \quad \text { eq. } 8 \\
=1+\frac{1}{2} C \cdot \exp \left(-k_{\text {kinetics }} \cdot \Delta \tau\right) \quad \text { eq. } 9
\end{gathered}
$$

With monoexponential fits using equation 9 we can extract the bunching amplitude $C$ and correlation relaxation rate $k_{\text {kinetics, }}$ which are listed for the two excitation intensities in Table S2.

Table S2: Monoexponential fit parameters from Figure S4d.

\begin{tabular}{c|cc}
\multicolumn{2}{c}{$\boldsymbol{C}$} & $\boldsymbol{k}_{\text {kinetics }} / \mathbf{s}^{-\mathbf{1}}$ \\
\hline $\mathbf{1 . 1} \mathbf{~ k W / \mathbf { c m } ^ { 2 }}$ & $0.614 \pm 0.004$ & $581 \pm 16$ \\
$\mathbf{4 . 1} \mathbf{~ k W / \mathbf { c m } ^ { 2 }}$ & $2.322 \pm 0.006$ & $1226 \pm 12$
\end{tabular}


With the extracted parameters we calculate the transition rates according to

$$
\begin{array}{ll}
k_{\text {on }}=\frac{k_{\text {kinetics }}}{C+1} & \text { eq. } 10 \\
k_{\text {off }}=\frac{k_{\text {kinetics }}}{\frac{1}{c}+1} & \text { eq. } 11
\end{array}
$$

The extracted rates are listed in Table S3.

\begin{tabular}{|c|c|c|}
\hline & $k_{\text {on }} / s^{-1}$ & $k_{\text {off }} / s^{-1}$ \\
\hline $1.1 \mathrm{~kW} / \mathrm{cm}^{2}$ & $359 \pm 9$ & $221 \pm 5$ \\
\hline $4.1 \mathrm{~kW} / \mathrm{cm}^{2}$ & $369 \pm 4$ & $856 \pm 8$ \\
\hline
\end{tabular}

Table S3: Calculated transition rate constants for two independently blinking dyes.

The rate constant for excursions into the on-state, $k_{\text {on }}$, stays constant within the error for both excitation intensities, as expected. However, the transition rate into the off-state, $k_{\text {off, }}$ is power dependent and shows a linear dependency of the excitation power. The power was raised by a factor of $\frac{4.1}{1.1} \sim 3.7$. The rate $k_{\text {off }}$ changed by a factor of $\frac{856}{221} \sim 3.8$, which supports our statement of a linear power dependency.

A fluorescence time trace of two collectively blinking ATTO 647N dyes is shown in Figure S5a. We started with the high excitation power of $4.1 \mathrm{~kW} / \mathrm{cm}^{2}$ and later switched to the lower excitation power of $1.1 \mathrm{~kW} / \mathrm{cm}^{2}$. At $123 \mathrm{~s}$, one ATTO $647 \mathrm{~N}$ dye bleached. Figure S5b and S5c show a two-second snapshot of fluorescence trajectories with $1 \mathrm{~ms}$ binning. The intensity histogram shows only two intensity states for both excitation powers. The ATTO 700 FRET-switch is constantly switching between a reduced state and the singlet manifold. In the reduced state, the absorption spectrum is blueshifted and the ATTO $647 \mathrm{~N}$ fluorescence is not quenched. When the ATTO 700 dye is oxidized again, the ATTO $647 \mathrm{~N}$ dyes are quenched by efficient FRET. Because we are monitoring the donor fluorescence, the bunching amplitude is inversely proportional to the excitation power. The donor dyes are stabilized by the ROXS buffer. The acceptor dye is mainly excited by FRET from the donor dyes. From its first excited energy state, it has a small chance to enter the long-living triplet state from which it can be reduced by Trolox. The time spent in the reduced state only depends on the Trolox quinone concentration and is independent of the excitation power. This fact is reflected in the intensity histograms of Figure S5b and S5c. For higher excitation power, the ATTO 647N dyes spend more time in the bright emitting state because they shelve the acceptor dye faster in the reduced state. On the other hand, with low excitation power, the donor dyes spend more time in the quenched emitting state because it takes longer to reduce the acceptor dye. 

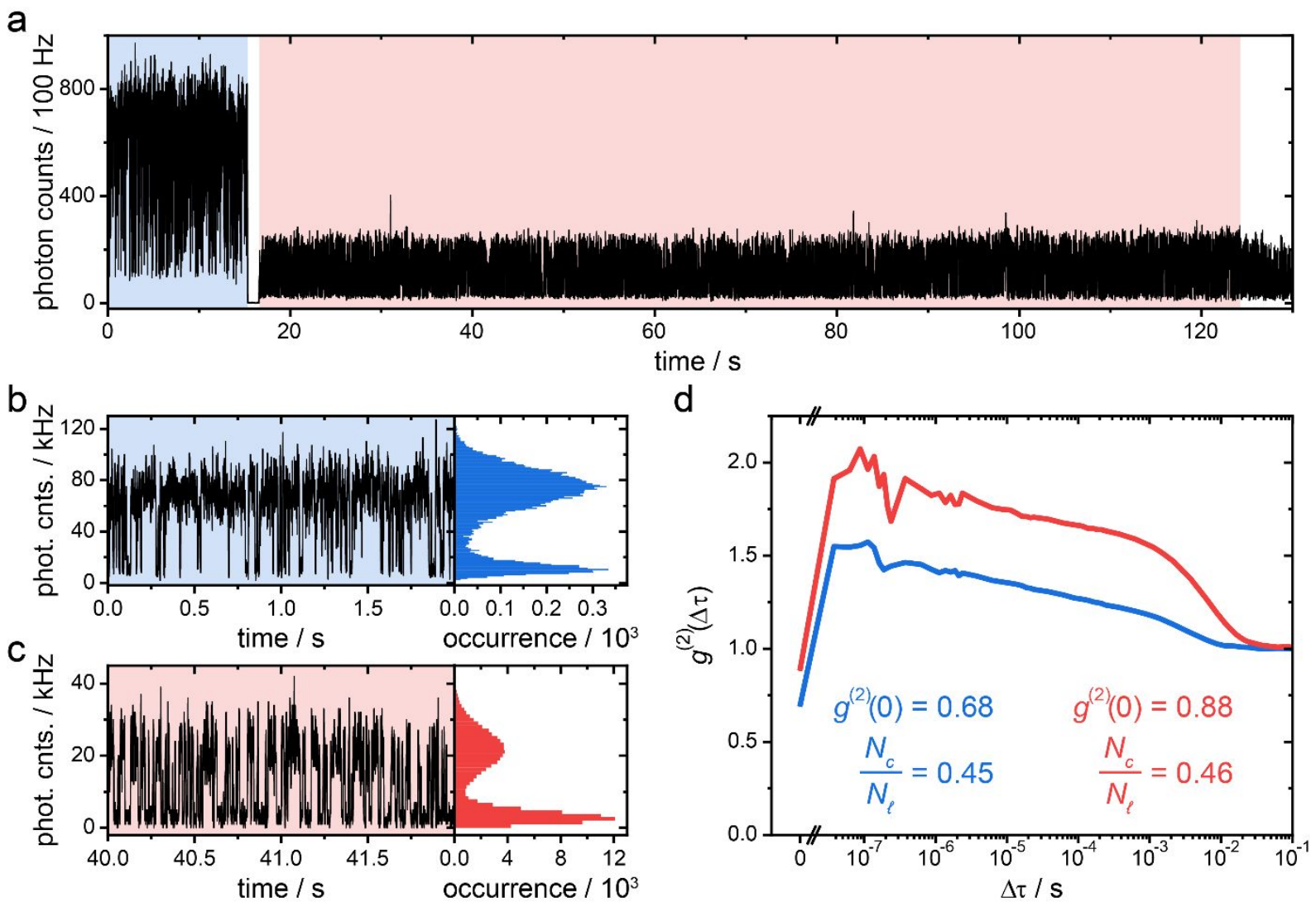

Figure S5. (a) Fluorescence time trace of two collectively blinking ATTO $647 \mathrm{~N}$ dyes modulated by an ATTO 700 FRET-switch. The excitation power was either $4.1 \mathrm{~kW} / \mathrm{cm}^{2}$ (blue) or $1.1 \mathrm{~kW} / \mathrm{cm}^{2}$ (red). (b) Two-second snippet of the fluorescence time trace at $4.1 \mathrm{~kW} / \mathrm{cm}^{2}$ excitation power and the corresponding intensity histogram. (c) Two-second snippet of the fluorescence trajectory at $1.1 \mathrm{~kW} / \mathrm{cm}^{2}$ excitation power and the corresponding intensity histogram. (d) Cross correlation of the fluorescence time traces.

\section{Change of excitation power does not correlate with a change in bunching amplitude}

As an example of blinking kinetics which does not show a change of the correlation amplitude for different excitation powers, we chose two blinking ATTO $647 \mathrm{~N}$ which are separated by $\sim 3 \mathrm{~nm}$, i.e. by 9 base pairs. The structure was published by Schröder et al. ${ }^{4}$ The dyes are not independent and interact by singlet-singlet annihilation (SSA) and singlet-dark-state annihilation (SDA). To promote the dye blinking, we removed enzymatic oxygen and excited the dyes at either $1.1 \mathrm{~kW} / \mathrm{cm}^{2}$ and $4.1 \mathrm{~kW} / \mathrm{cm}^{2}$. We did not add Trolox to have sufficient singlet-triplet interactions. The fluorescence time trace for both excitation powers is shown in Figure S6a. The correlation amplitude for a single dye usually scales linearly with the excitation power. For two dyes with weak coupling this statement is not true anymore as demonstrated in Figure S6b. The correlation amplitude stays constant, but the correlation relaxation time is shifted to shorter timescales with increasing excitation power. Due to SSA and SDA, the excitation into higher excited states becomes more likely. In higher excited states, the transition rates between the singlet and triplet state are larger and the blinking kinetics becomes faster. In our case, the rates scale with the same factor, although it varies for different dye combinations. For the antibunching measurement, we obtain $g^{(2)}(0) \sim 0.3$ and $N_{\mathrm{c}} / N_{\ell} \sim 0.2$, which is expected for weakly collectively blinking dyes when they undergo efficient SSA.

When one dye enters the dark state, it quenches the second dye by a FRET mechanism referred to as SDA. This results in a shorter fluorescence lifetime and a quenched fluorescence signal. As described in section S5, changes of the correlation amplitude for different microtime gates shown in Figure S6c, d 
are a characteristic feature of collectively blinking dyes. Therefore, this correlation amplitude can be assigned to collective blinking kinetics without changing the excitation power, and the normalization of photon antibunching has to be carried out with respect to the bunching amplitude.
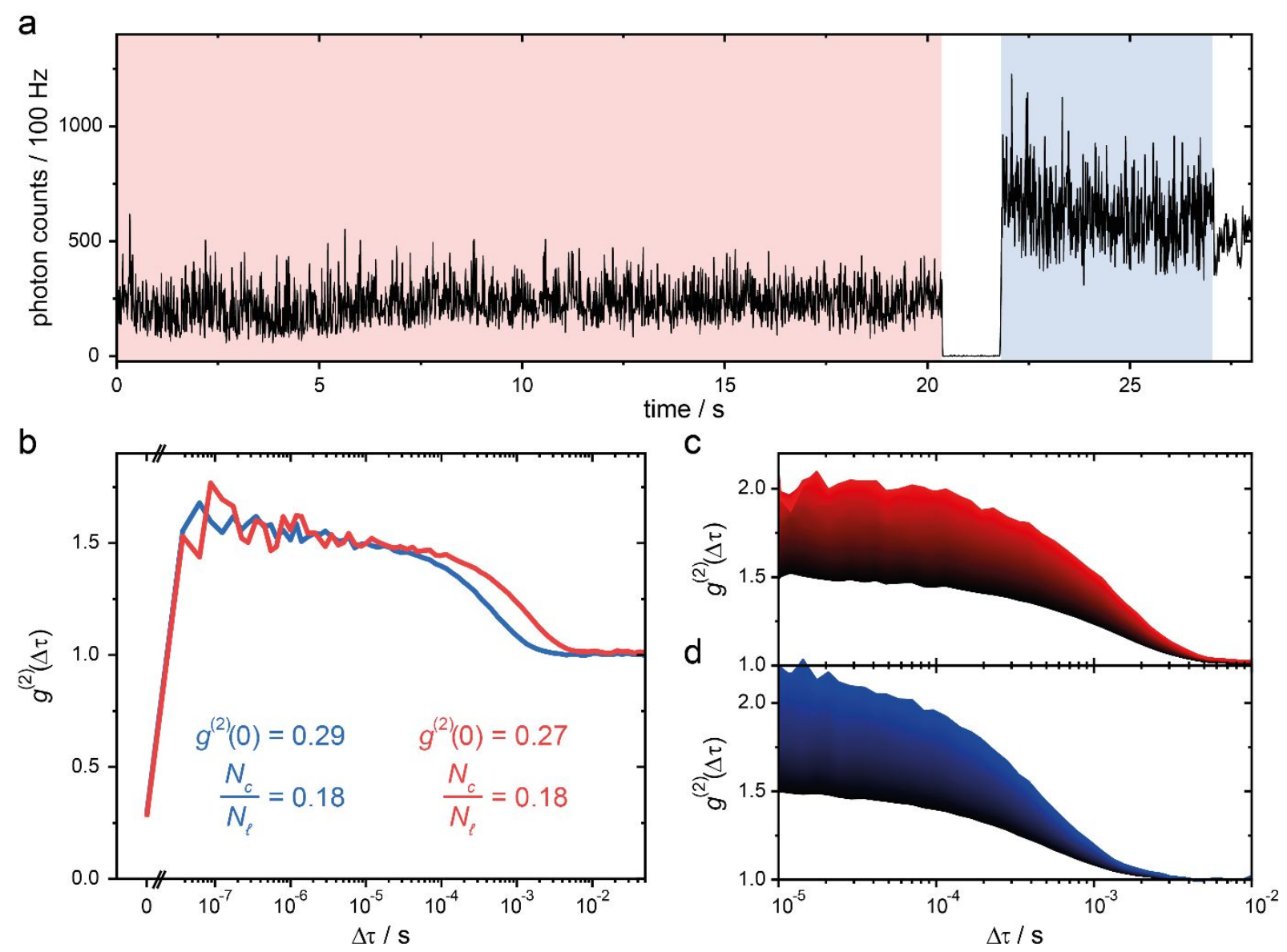

Figure S6. (a) Fluorescent trajectory of two collectively blinking ATTO $647 \mathrm{~N}$ dyes separated by $3 \mathrm{~nm}$. The excitation power was switched from $1.1 \mathrm{~kW} / \mathrm{cm}^{2}$ (red) to $4.1 \mathrm{~kW} / \mathrm{cm}^{2}$ (blue). (b) Cross-correlation of the fluorescence time traces for either excitation powers. (c) Cross-correlation for the $1.1 \mathrm{~kW} / \mathrm{cm}^{2}$ excitation power. The color gradient marks the beginning of the microtime gate used, which was shifted with a $20 \mathrm{ps}$ step size. Black corresponds to early (from $0 \mathrm{~ns}$ ) gate times and red to late times (up to $6 \mathrm{~ns}$ ). (d) Cross-correlation for the $4.1 \mathrm{~kW} / \mathrm{cm}^{2}$ excitation power. The color gradient marks the beginning of the microtime gate, which was shifted in $20 \mathrm{ps}$ step sizes. Black corresponds to early (from $0 \mathrm{~ns}$ ) gate times and blue to late times (up to $6 \mathrm{~ns}$ ).

\section{Impact of simultaneous excitation of two chromophores}

The corresponding average fluorescence signals of a single chromophore are $15 \mathrm{kHz}$ and $58.5 \mathrm{kHz}$, respectively, which corresponds to a linear increase for $1.1 \mathrm{~kW} / \mathrm{cm}^{2}$ and $4.3 \mathrm{~kW} / \mathrm{cm}^{2}$ excitation irradiance as shown in Figure S4. The excitation irradiance is derived by measuring the power at the back aperture of the objective, which is focused onto a diffraction-limited excitation spot. A short estimation by using the detection rate is given in the following. Two independently blinking chromophores yield, on average, a signal of $30 \mathrm{kHz}$ at $1.1 \mathrm{~kW} / \mathrm{cm}^{2}$ excitation power, and by estimating a detection efficiency of $20 \%$ this signal corresponds to 75,000 photons emitted per second per molecule. With a fluorescence quantum yield of $65 \%$ we would excite the molecule $\sim 115,000$ times per second. The pulsed excitation rate of our laser is set to $40 \mathrm{MHz}$, which means that we excite the chromophore, on average, with every $348^{\text {th }}$ pulse. If we were to have perfect singlet-singlet annihilation between two chromophores, we would lose one fluorescence photon for every $348 \times 348$ excitation 
pulses. This loss corresponds to 331 excitons per second. Taking into account the detection efficiency of the microscope, this means that $\sim 43$ photons per second are lost. The intensity would therefore drop by only $0.3 \%$. The same calculation yields an intensity drop of $\sim 1.1 \%$ for $4.3 \mathrm{~kW} / \mathrm{cm}^{2}$ excitation intensity.

\section{Change of photon bunching with microtime gating}

Random switching between two intensity states $I_{\mathrm{A}}$ and $I_{\mathrm{B}}$ is depicted in Figure $\mathrm{S} 7 \mathrm{a}$. The corresponding $g^{(2)}(\Delta \tau)$ intensity correlation is depicted in Figure S7b.
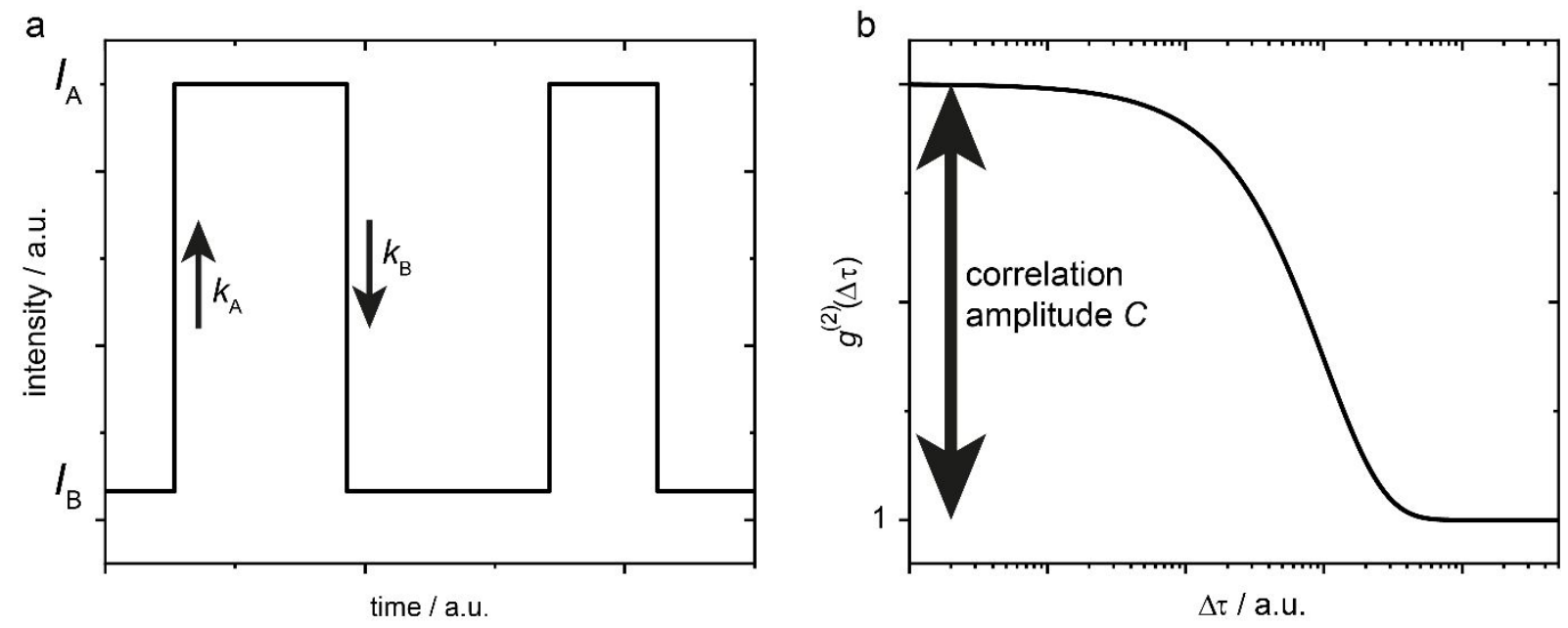

Figure S7. (a) Variation of the fluorescence intensity of a blinking molecule. (b) The corresponding correlation function on a logarithmic time scale. The correlation amplitude $C$ is highlighted.

The correlation amplitude $C$ of a system with two intensity states is given as: ${ }^{5-8}$

$$
C=K\left(\frac{I_{\mathrm{A}}-I_{\mathrm{B}}}{I_{\mathrm{A}}+K \cdot I_{\mathrm{B}}}\right)^{2} \quad \text { eq. } 12
$$

where $I_{\mathrm{A}}$ is the intensity of the bright fluorescent state $\mathrm{A}$ and $I_{\mathrm{B}}$ represents the intensity of the quenched fluorescent state B. $K$ is the equilibrium constant calculated from the rate $k_{\mathrm{B}}$ of $\mathrm{A} \rightarrow \mathrm{B}$ state transitions and the rate $k_{\mathrm{A}}$ of $\mathrm{B} \rightarrow \mathrm{A}$ state transitions.

$$
K=\frac{k_{\mathrm{B}}}{k_{\mathrm{A}}} \quad \text { eq. } 13
$$

The correlation amplitude $C$ relies on the fractional intensity difference between states $\mathrm{A}$ and $\mathrm{B}$. The quenched state $B$ has an additional non-radiative decay from the electronic excited state. Therefore, the fluorescence decays faster after excitation in the quenched state which changes $C$ for different microtime gates.

In our approach we vary the beginning of the microtime gate $t$, i.e. we use a long-pass time gate to change $C(t)$. We integrate over the exponential decay of the excited state from $t$ to infinity to calculate the intensity of the quenched and unquenched state, respectively:

$$
\begin{aligned}
& I_{\mathrm{A}}(t)=\int_{t}^{\infty} e^{-\left(k_{\mathrm{r}}+k_{\mathrm{nr}}\right) \cdot t^{\prime}} d t^{\prime}=\frac{e^{-\left(k_{\mathrm{r}}+k_{\mathrm{nr}}\right) \cdot t}}{k_{\mathrm{r}}+k_{\mathrm{nr}}}, \quad \text { eq. } 14 \\
& I_{\mathrm{B}}(t)=\int_{t}^{\infty} e^{-\left(k_{\mathrm{r}}+k_{\mathrm{nr}}+k_{\mathrm{SDA}}\right) \cdot t^{\prime}} d t^{\prime}=\frac{e^{-\left(k_{\mathrm{r}}+k_{\mathrm{nr}}+k_{\mathrm{SDA}}\right) \cdot t}}{k_{\mathrm{r}}+k_{\mathrm{nr}}+k_{\mathrm{SDA}}} . \quad \text { eq. } 15
\end{aligned}
$$

Here, $k_{\mathrm{r}}$ and $k_{\mathrm{nr}}$ are the radiative and non-radiative decay rates of state $\mathrm{A}$, respectively. $k_{\mathrm{SDA}}$ is the additional SDA rate present for state B. This leads to: 


$$
C(t)=K\left(\frac{\frac{e^{-\left(k_{\mathrm{r}}+k_{\mathrm{nr}}\right) \cdot t}}{k_{\mathrm{r}}+k_{\mathrm{nr}}}-\frac{e^{-\left(k_{\mathrm{r}}+k_{\mathrm{nr}}+k_{\mathrm{SDA}}\right) \cdot t}}{k_{\mathrm{r}}+k_{\mathrm{nr}}+k_{\mathrm{SDA}}}}{\frac{e^{-\left(k_{\mathrm{r}}+k_{\mathrm{nr}}\right) \cdot t}}{k_{\mathrm{r}}+k_{\mathrm{nr}}}+K \cdot \frac{e^{-\left(k_{\mathrm{r}}+k_{\mathrm{nr}}+k_{\mathrm{SDA}}\right) \cdot t}}{k_{\mathrm{r}}+k_{\mathrm{nr}}+k_{\mathrm{SDA}}}}\right)^{2}=K\left(\frac{1-\frac{k_{\mathrm{r}}+k_{\mathrm{nr}}}{k_{\mathrm{r}}+k_{\mathrm{nr}}+k_{\mathrm{SDA}}} e^{-k_{\mathrm{SDA}} \cdot t}}{1+K \cdot \frac{k_{\mathrm{r}}+k_{\mathrm{nr}}}{k_{\mathrm{r}}+k_{\mathrm{nr}}+k_{\mathrm{SDA}}} e^{-k_{\mathrm{SDA}} \cdot t}}\right)^{2} \quad \text { eq. } 16
$$

The contrast between the integrated intensities rises with the additional depletion rate $k_{\mathrm{SDA}}$. Equation 16 shows that $C(t)$ depends exponentially on $k_{\mathrm{SDA}}$. We assume that $k_{\mathrm{r}}$ and $k_{\mathrm{nr}}$ stay constant and we are only interested in the values of $K$ and $k_{\mathrm{SDA}}$. Therefore, we can rewrite eq. 5 as

$$
C(t)=K\left(\frac{1-e^{-k_{\mathrm{SDA}} \cdot\left(t-t_{0}\right)}}{1+K \cdot e^{-k_{\mathrm{SDA}} \cdot\left(t-t_{0}\right)}}\right)^{2}, \quad \text { eq. } 17
$$

where $t_{0}$ is an artificial shift on the time axis that accounts both for the arrival time of the laser pulse and the fluorescence lifetime ratio $\frac{k_{\mathrm{r}}+k_{\mathrm{nr}}}{k_{\mathrm{r}}+k_{\mathrm{nr}}+k_{\mathrm{SDA}}}$.

To demonstrate that this approach allows extraction of $k_{\mathrm{SDA}}$ from measured data, we simulated two simultaneously blinking chromophores similar to the experimental situation. The switching rates were fixed to $k_{\mathrm{A}}=k_{\mathrm{B}}=10^{4} \mathrm{~s}^{-1}$ and the fluorescence lifetimes were chosen to be $4 \mathrm{~ns}$ for the bright and $1 \mathrm{~ns}$ for the quenched state. Therefore, the additional non-radiative rate for quenched state is $k_{\mathrm{SDA}}=\frac{1}{\tau_{\mathrm{B}}}-\frac{1}{\tau_{\mathrm{A}}}$ $=0.75 \mathrm{~ns}^{-1}$. Figure S8a shows the corresponding $g^{(2)}(\Delta \tau)$ correlation for different starting points $t$ of the microtime gates, which were shifted in steps of $20 \mathrm{ps}$. Due to the increasing contrast in the intensity levels, the autocorrelation amplitude rises with $t$. Figure S8b shows $g^{(2)}\left(\Delta \tau=10^{-8} \mathrm{~s}\right)$ in red, which in our case is equivalent to the correlation amplitude $C(t)$ corrected by an offset of 1 for uncorrelated events. We fit the extracted value with

$$
g^{(2)}\left(\Delta \tau=10^{-8} S, t\right)=1+K\left(\frac{1-e^{-k_{\mathrm{SDA}} \cdot\left(t-t_{0}\right)}}{1+K \cdot e^{-k_{\mathrm{SDA}} \cdot\left(t-t_{0}\right)}}\right)^{2} \quad \text { eq. } 18
$$

The value of $k_{\mathrm{SDA}}$ extracted from the fit (bold semitransparent line) is $k_{\mathrm{SDA}}=0.748 \pm 0.002 \mathrm{~ns}^{-1}$, close to the expected value of 0.75 . Additionally, we plot $g^{(2)}(\Delta \tau=0, t)$ in black, which shows a saturation towards 1 corresponding to an apparently infinite number of chromophores. The ratio of $g^{(2)}(\Delta \tau=0, t)$ and $g^{(2)}\left(\Delta \tau=3 \cdot 10^{-8} \mathrm{~s}, t\right)$ equals the $N_{\mathrm{c}} / N_{\ell}$ ratio and stays constant at a value of 0.5 , the expected value for a system with two chromophores.
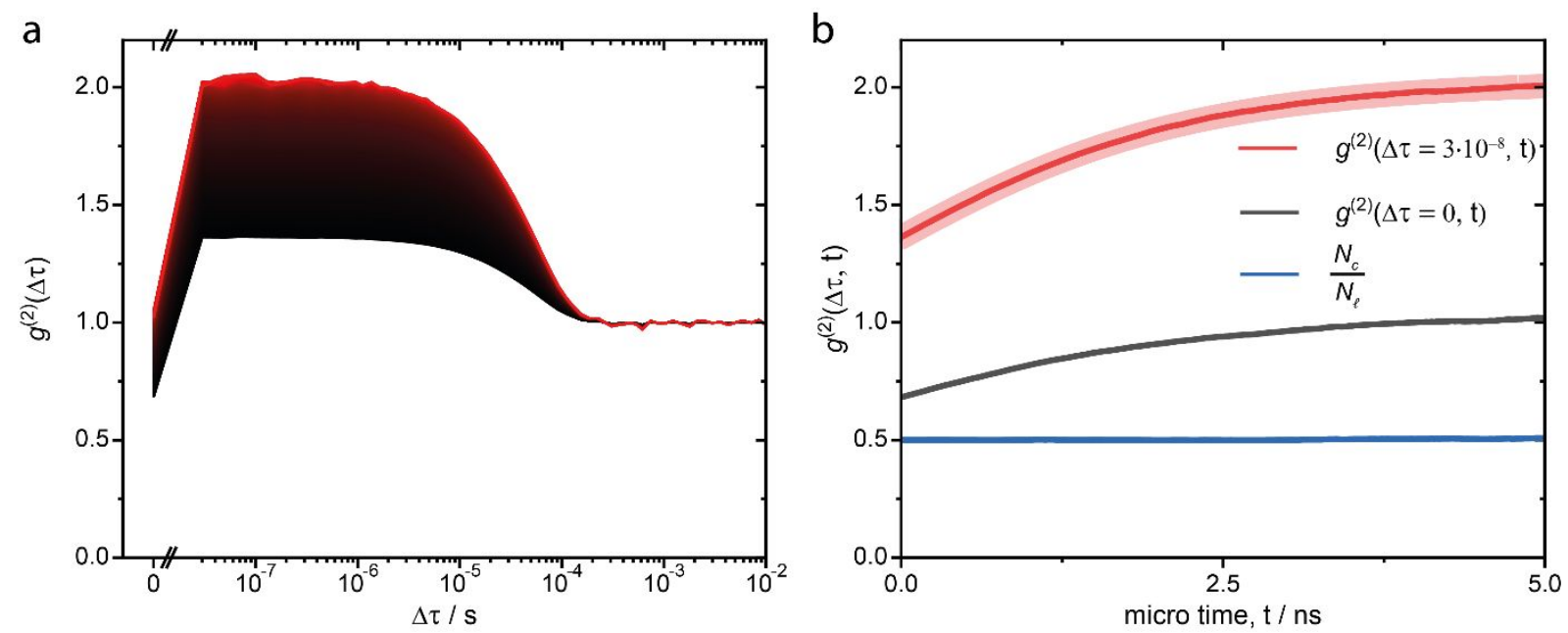

Figure S8. (a) Simulated correlation of two simultaneously blinking dyes. The color gradient marks the beginning of the microtime gate used, which was shifted with a $20 \mathrm{ps} \mathrm{step} \mathrm{size.} \mathrm{Black} \mathrm{corresponds} \mathrm{to} \mathrm{an}$ early (from $0 \mathrm{~ns}$ ) beginning of the microtime gate and red to a late one (up to $5 \mathrm{~ns}$ ). (b) Extracted 
correlation values for $g^{(2)}\left(\Delta \tau=10^{-8} s, t\right)$ (red) and $g^{(2)}(\Delta \tau=0, t)$ (black). The red data set is fitted with eq. 7 (semitransparent bold red line). The ratio of $N_{\mathrm{c}} / N_{\ell}$ is plotted in blue.

To extract the $k_{\mathrm{SDA}}$ rate from our experimental data we plotted the blinking amplitude of Figure $4 \mathrm{j}$ with a mono-exponential model to extract $C(t)$ for different beginning values of the microtime gate.

$$
g^{(2)}(\Delta \tau, t)=1+C(t) \cdot \exp \left(-\left(k_{\text {kinetics }}\right) \cdot \Delta \tau\right) \quad \text { eq. } 19
$$

Here, $k_{\text {kinetics }}=k_{\mathrm{A}}+k_{\mathrm{B}}$ is the sum of the kinetic rates that describe the state transitions. The correlation amplitude for different microtime gates is shown in Figure S9a. The extracted correlation amplitudes $C$ $(t)$ are plotted in Figure $\mathrm{S} 9 \mathrm{~b}$ as a black line and fitted with eq. 6 . We extract $k_{\mathrm{SDA}}$ from the fit as $k_{\mathrm{SDA}}$ $=1.41 \pm 0.01 \mathrm{~ns}^{-1}$.
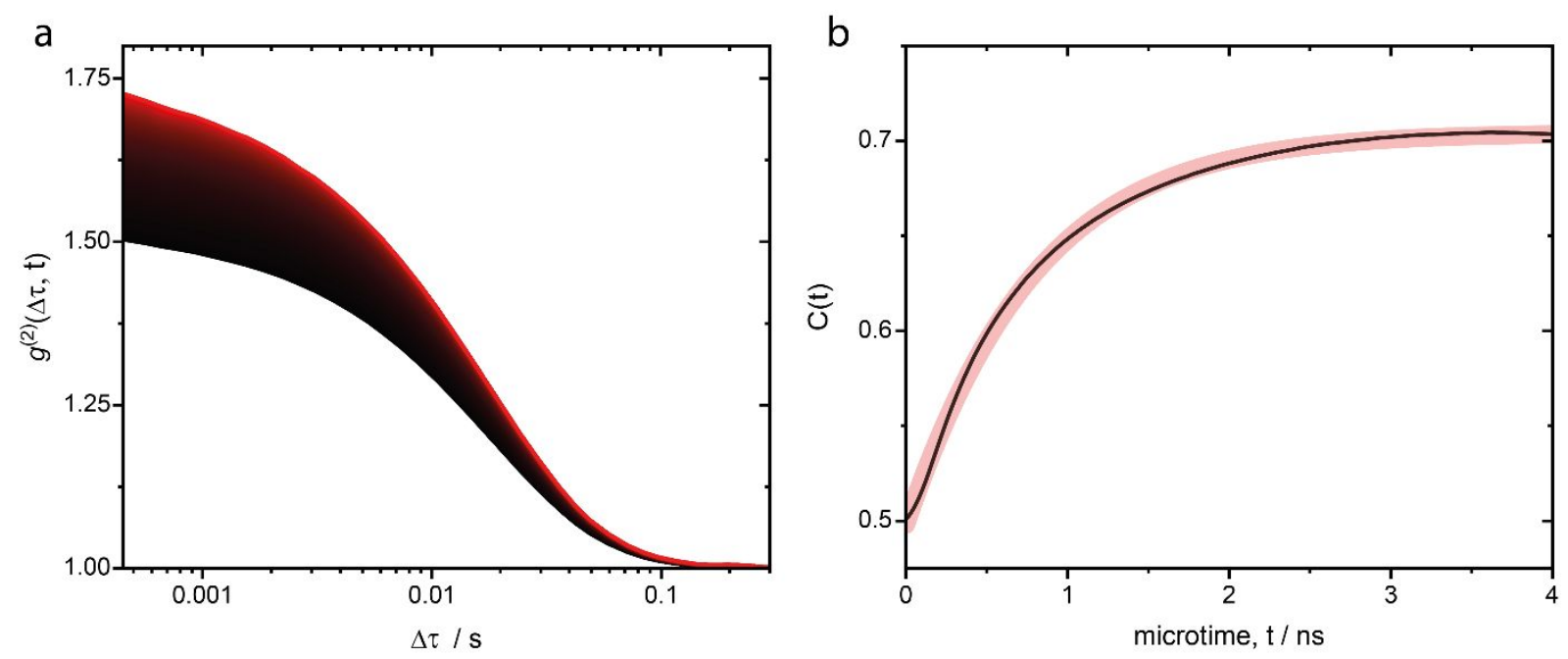

Figure S9. (a) Correlation amplitude of two collectively blinking ATTO $647 \mathrm{~N}$ dyes. The color gradient marks the beginning of the microtime gate used, which was shifted in steps of $20 \mathrm{ps}$. Black corresponds to an early beginning (from $0 \mathrm{~ns}$ ) of the microtime gates and red to a late (up to $4 \mathrm{~ns}$ ) beginning. (b) Fitted correlation amplitude $C(t)$ shown in black, with a fit based on eq. 6 shown as a red semitransparent line.

\section{Fluorescence lifetime fitting and SDA rate extraction}

A reconvolution algorithm was used in order to extract the fluorescence lifetimes from the photon arrival time histograms in Figure $4 \mathrm{~g}$ and $4 \mathrm{~h}$. The fluorescence lifetime decay was fitted by a convolution of the instrument response function $\left(I_{\mathrm{IRF}}\right)$ and the sum of fluorescence lifetime decays with amplitudes $A_{i}$, fluorescence lifetime $\tau_{\mathrm{fl}, i}$ and the background intensity $I_{\mathrm{bg}}$.

$$
I(t)=\left(I_{\mathrm{IRF}}(t) * \sum_{i} A_{i} \cdot e^{-\frac{t-t_{\mathrm{off}}}{\tau_{\mathrm{fl}, i}}}\right)+I_{\mathrm{bg}} \quad . \quad \text { eq. } 20
$$

Here, $*$ denotes the convolution operator and $t_{\text {off }}$ represents an offset of the decay function that was introduced to compensate an intensity-dependent shift of $I_{\mathrm{IRF}}$. The fit routine iterates over a range of IRF shifts and returns the fit attempt with the best reduced chi-squared test. The reconvolution fits are shown in Figure S10. The monoexponential fit model in Figure S10a works well for the independently blinking chromophore data of Figure 4i. The reduced chi squared is 1.05. A fluorescence lifetime of $4.3 \pm 0.1 \mathrm{~ns}$ was extracted, which is expected for ATTO $647 \mathrm{~N}$ labeled on dsDNA. ${ }^{9}$ The monoexponential model works rather poorly for collectively blinking chrompohores as shown in Figure S10b, with a reduced chi squared of 9.72. A biexponential model is assumed to better model the presence of two intensity states of the dyes. The corresponding reconvolution fit results in reduced chi-squared 
values of 1.62 and the obtained lifetimes are $\tau_{\mathrm{fl}, 1}=0.6 \pm 0.1 \mathrm{~ns}$ and $\tau_{\mathrm{fl}, 2}=2.7 \pm 0.1 \mathrm{~ns}$. The slow component shows that the ATTO 700 still absorbs slightly in its anionic radical state.
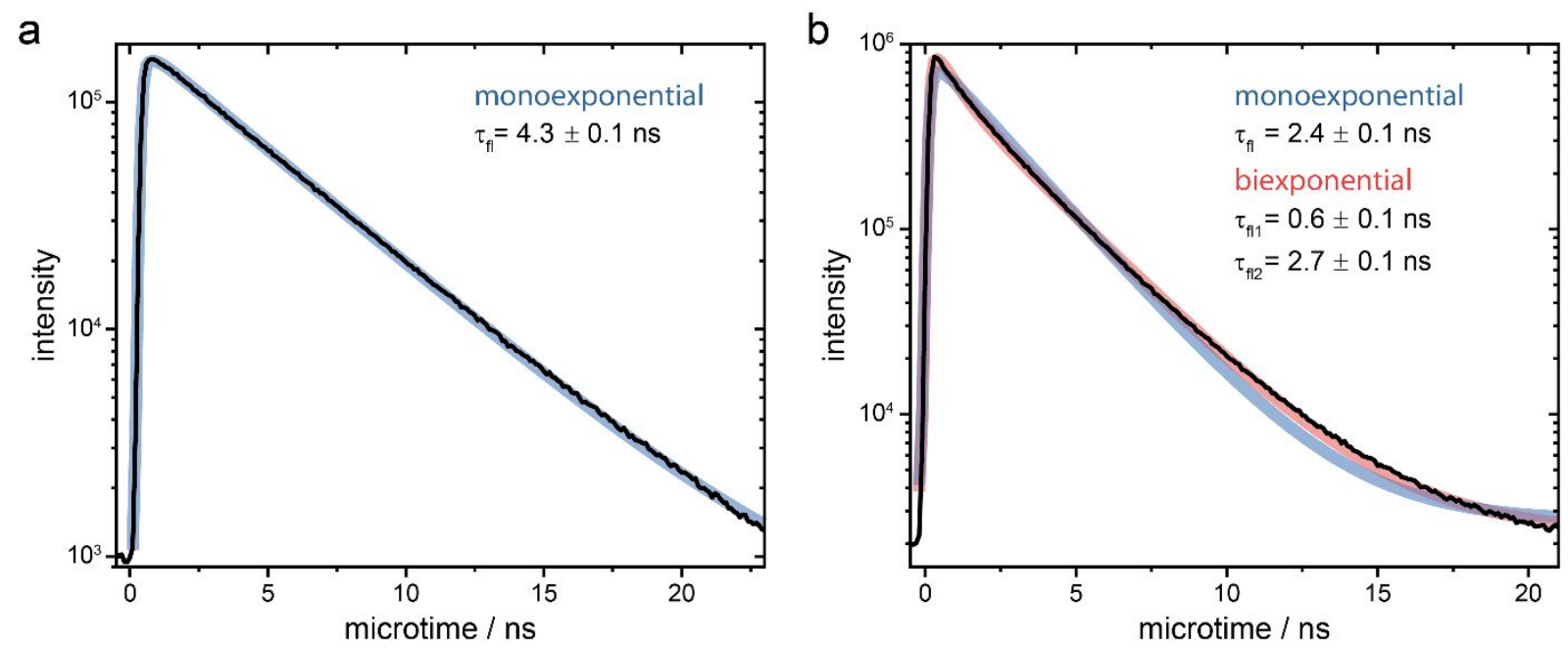

Figure S10. (a) Monoexponential reconvolution fit (blue) of the data from Figure 4g (black) with a microtime binning of 100 ps. (b) Monoexponential (blue) and biexponential (red) reconvolution fits of the data from Figure $4 \mathrm{~h}$ (black) with a microtime binning of $100 \mathrm{ps}$.

With the fluorescence lifetimes extracted from the biexponential fit model we are able to calculate the SDA rate and validate our method as discussed in section 5.

$$
\frac{1}{\tau_{\mathrm{fl}, 1}}-\frac{1}{\tau_{\mathrm{fl}, 2}}=\frac{1}{\frac{1}{k_{\mathrm{r}}+k_{\mathrm{nr}}+k_{\mathrm{SDA}}}}-\frac{1}{\frac{1}{k_{\mathrm{r}}+k_{\mathrm{nr}}}}=k_{\mathrm{SDA}} \quad \text { eq. } 21
$$

This approach yields $k_{\mathrm{SDA}}=1.3 \pm 0.3 \mathrm{~ns}^{-1}$, which is in good agreement with the SDA rate extracted in section 5 of $k_{\mathrm{SDA}}=1.41 \pm 0.01 \mathrm{~ns}^{-1}$.

\section{Systems with simultaneous collective and independent blinking}

Multichromophoric systems could potentially show a combination of collective and independent blinking of chromophores. Both types of blinking require different normalizations of the photon antibunching measurement and therefore neither $g^{(2)}(0)$ nor $N_{\mathrm{c}} / N_{\ell}$ yields the correct number of chromophores. Here, we demonstrate by simulations how a suitable subset of fluorescence photons can yield the correct value.

For the simulations we consider a simple two-chromophore system. Two chromophores are collectively quenched by an energy sink. In Figure S11, the collective switching of two chromophores into the quenched and unquenched state is denoted by the transition rate constants $k_{\mathrm{B}}$ and $k_{\mathrm{A}}$, respectively. The independent blinking of the dyes by switching between a florescent and non-fluorescent state is denoted with $k_{\text {off }}$ and the transition rate into the fluorescent state with $k_{\text {on }}$.

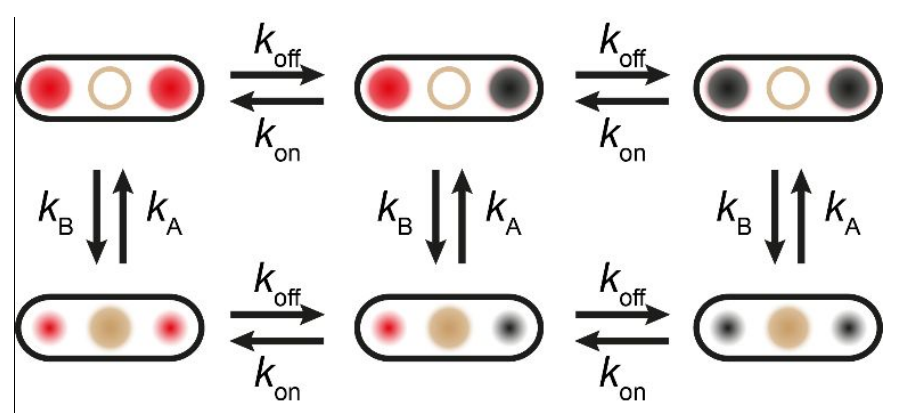


Figure S11. Sketch of all five intensity states of a system with two independently blinking dyes which can also get collectively quenched by an energy sink. We note that both states on the right are considered as one state because both states are completely non-fluorescent.

The simulations were carried out as described in Section 2. The transition rates for Figure S12a,b are set to: $k_{\mathrm{A}}=k_{\mathrm{B}}=500 \mathrm{~s}^{-1}$ and $k_{\mathrm{on}}=k_{\mathrm{off}}=50000 \mathrm{~s}^{-1}$. The transition rates for Figure S12c,d are set to: $k_{\mathrm{A}}$ $=k_{\mathrm{B}}=50000 \mathrm{~s}^{-1}$ and $k_{\mathrm{on}}=k_{\mathrm{off}}=500 \mathrm{~s}^{-1}$.
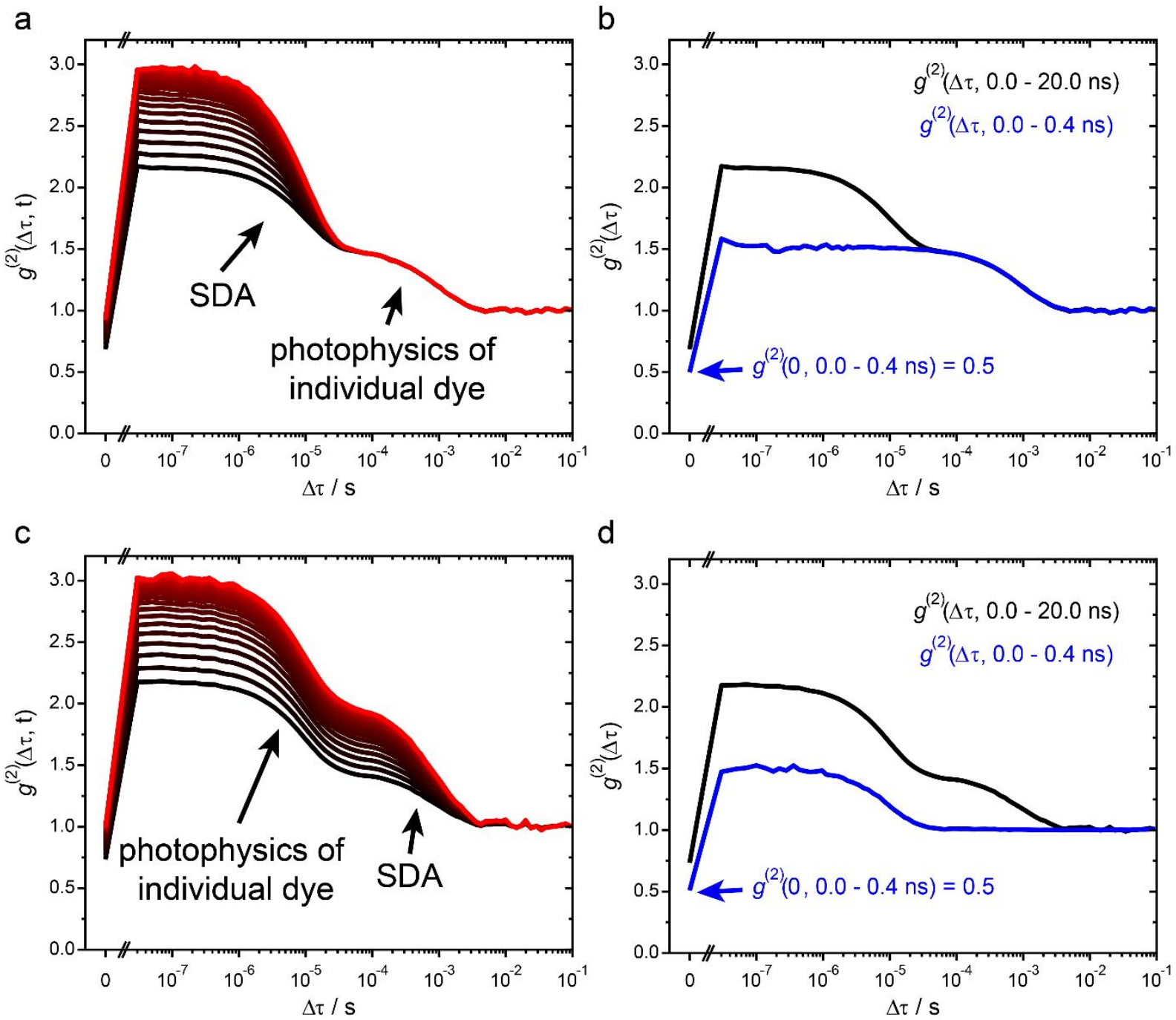

Figure S12. (a, c) Simulated intensity correlation of two collectively blinking dyes, which also show individual blinking. The color gradient marks the beginning of the microtime gate $t$ used, which was shifted in steps of $200 \mathrm{ps}$. Black corresponds to an early (from $0 \mathrm{~ns}$ ) beginning of the microtime gate and red to a late one (up to $5 \mathrm{~ns}$ ). (b, d) Intensity correlation curves considering all photons (black) and only the photons detected within the first 400 ps after pulsed excitation (blue). Note that the bunching amplitude due to collective blinking is missing and that $g^{(2)}(0)$ yields the correct degree of photon antibunching.

The expected antibunching value for two dyes is 0.5 . The simulation with faster collective blinking (SDA, Figure S12a) kinetics yields a degree of photon antibunching of $g^{(2)}(0)=0.69$ and $N_{\mathrm{c}} / N_{\ell}$ $=0.32$. The simulation with faster individual blinking (Figure S12c) yields a degree of photon antibunching of $g^{(2)}(0)=0.73$ and $N_{\mathrm{c}} / N_{\ell}=0.34 . g^{(2)}(0)$ overestimates the number of dyes because collective blinking is present, which raises the $g^{(2)}(0)$ value. $N_{\mathrm{c}} / N_{\ell}$ underestimates the number of dyes because the lateral correlation bins are now the sum of the two bunching amplitudes but only the amplitude of the collective blinking is affecting the zero-lag-time correlation $g^{(2)}(0)$. 
An easy approach to count the number of chromophores is the application of a small microtime gate right after the pulsed excitation. The quenching of the SDA is a time dependent process and becomes more likely as time progresses, which ultimately results in a quenched fluorescence intensity and shortened fluorescence lifetime. If there were only little time for SDA processes, both intensity levels would be equally bright if only photons right after the laser pulse excitation are considered. Within the first few hundred picoseconds no intensity fluctuations due to SDA are observed, resulting in an absence of bunching amplitude in the intensity correlation as depicted by the blue lines in Figure S12b,d. The remaining bunching amplitude results from the independent blinking process and therefore the number of chromophores can be reliably counted by $g^{(2)}(0)$ as demonstrated in Figure S12b,d. Choosing an early time gate is also useful if SSA has to be considered, which is also a time dependent process. ${ }^{9}$ With an early microtime gate annihilation processes have most likely not occurred yet and no excitons are lost due to SSA nor SDA.

Table S4: Sequences for the modified DNA origami structure.

\begin{tabular}{|c|c|c|}
\hline $5^{6}$ position & Sequence & Comment \\
\hline $0[286]$ & AAAACGAAAGAGGCTCATTATAC & \\
\hline $11[105]$ & ACACAACATACGAGGGATGTGGCTATTAATCGGCC & \\
\hline $9[567]$ & ATCATTTACATAAAAGTATCAAAATTATAAGAAACTTCAATA & \\
\hline $7[567]$ & CAGCTTTGAATACCAAGTTACAA & \\
\hline $5[455]$ & CATGCCAGTGAGCGCTAATATCCAATAATAAGAGC & \\
\hline $2[223]$ & CCGAACTTTAATAAAAGCAAAGCGGATT & \\
\hline $5[497]$ & TTGAGAATATCTTTCCTTATCACTCATCGAGAACA & \\
\hline $9[315]$ & CAGATATAGGCTTGAACAGACGTTAGTAAAGCCCAAAAATTT & \\
\hline $5[287]$ & GCGCAGCGACCAGCGATTATATATCATCGCCTGAT & \\
\hline $8[69]$ & TCGGTCATACCGGGGGTTTCTGC & \\
\hline $11[219]$ & GTGCCTGCTTTAAACAGGGAGAGAGTTTCAAAGCGAACCA & \\
\hline $10[457]$ & AAAAGATAGGGTTGAGTGT & \\
\hline $2[643]$ & GATAGTGCAACATGATATTTTTGAATGG & \\
\hline $0[347]$ & AGCGTATCATTCCACAGACCCGCCACAGTTGCAGCAAGCG & \\
\hline $9[483]$ & ATAATGAATCCTGAGATTACGAGCATGTGACAAAAACTTATT & \\
\hline $8[573]$ & AAATGCGTTATACAAATTCTTAC & \\
\hline $2[433]$ & AGGGACAAAATCTTCCAGCGCCAAAGAC & \\
\hline $7[63]$ & GCCCGCACAGGCGGCCTTTAGTG & \\
\hline $4[377]$ & CTATTTCGGAACGAGTGAGAATA & \\
\hline $0[698]$ & TTTTTCGGGAGCTAAACAGGTTGTTAGAATCAGAGTTTTT & \\
\hline $4[587]$ & CATCGGGAGAAATTCAAATATAT & \\
\hline $7[506]$ & AAATCAGCCAGTAATAACACTATTTTTGAAGCCTTAAATC & \\
\hline $7[170]$ & TTTTTATCCAATAAATCTCTACCCCGGTAAAACTAGCATG & \\
\hline $5[161]$ & GTATACAGGTAATGTGTAGGTAGTCAAATCACCAT & \\
\hline $4[396]$ & AACAGAGTGCCTGGGGTTTTGCTCACAGAAGGATTAGGAT & \\
\hline $3[350]$ & GTCACCAGTACAAGGTTGAGGCA & \\
\hline $5[581]$ & ACATCATTTAAATTGCGTAGAAACAGTACCTTTTA & \\
\hline $5[623]$ & ATACCCTTCGTGCCACGCTGAACCTTGCTGAACCT & \\
\hline $8[130]$ & GGGCGTGAAATATTAGCGCCATTCGC & \\
\hline $9[357]$ & TCTTATACTCAGAAAGGCTTTTGATGATATTGACACGCTATT & \\
\hline $11[345]$ & GAGAGCCTCAGAACCGCATTTTCTGTAACGATCTAAAGTT & \\
\hline
\end{tabular}




\begin{tabular}{|c|c|}
\hline $5[329]$ & TTCATTTTCTGCTAAACAACTGAACAACTAAAGGA \\
\hline $8[489]$ & AAAACGGAATACCCAAAAGAACT \\
\hline $0[202]$ & GACCGGAAGCAATTGCGGGAGAA \\
\hline $3[182]$ & GCTAAATCGGTTTGACTATTATA \\
\hline $3[392]$ & ATATTCACAAACAAATTCATATG \\
\hline $6[69]$ & AAAAGTGTCAGCAACAATTGCAGGCGCT \\
\hline $11[567]$ & ACCATCACCCAAATAAACAGTTCATTTGATTCGCC \\
\hline $7[590]$ & AATCGTTGAGTAACATTGGAATTACCTAATTACATTTAAC \\
\hline $11[93]$ & GCTCAAGTTGGGTAACGGGCGGAAAAATTTGTGAGAGATA \\
\hline $0[305]$ & ACTACCTTTAAACGGGTAACAGGGAGACGGGCA \\
\hline $4[270]$ & TCAACATCAGTTAAATAGCGAGAGTGAGACGACGATAAAA \\
\hline $6[153]$ & TAAATCGGTTGGTGCACATCAAAAATAA \\
\hline $10[163]$ & TCAGCTAACTCACATTAAT \\
\hline $7[231]$ & TGCAACACTATCATAACCCTCGT \\
\hline $4[438]$ & ACCAAATTACCAGGTCATAGCCCCGAGTTTTCATCGGCAT \\
\hline $8[195]$ & TTAACAAGAGAATCGATGAACGG \\
\hline $3[625]$ & AGACAACCTGAACAGTATTCGAC \\
\hline $8[363]$ & TGAACAGCTTGATACCGATAGTT \\
\hline $0[412]$ & TCACCGTCACCGGCGCAGTCTCT \\
\hline $4[706]$ & TTTTTGTCCATCACGCAAATTCCGAGTAAAAGAGTCTTTTTT \\
\hline $11[315]$ & ACAGCTGATTGCCCGTCGCTGCGCCCACACGTTGA \\
\hline $8[424]$ & CGGAAGCACGCAAACTTATTAGCGTT \\
\hline $0[431]$ & ATTCAAGGGGAAGGTAAATGTGGCAAATAAATC \\
\hline $3[602]$ & TGATTATCAGATATACGTGGCAC \\
\hline $4[545]$ & TGACCTAAATTTTTAAACCAAGT \\
\hline $3[679]$ & GGTTGCTTTGACGAGCACGTTTTT \\
\hline $6[573]$ & TGATTTAGAAAACTCAAGAGTCAATAGT \\
\hline $11[441]$ & AAAAGAATAGCCCGATACATACGCAGTAAGCTATC \\
\hline $8[634]$ & TACATAAATTCTGGGCACTAACAACT \\
\hline $3[541]$ & CATAGTTAATTTGTAAATGTCGC \\
\hline $11[147]$ & TGCCTAATGAGTGAGAAAAGCTCATATGTAGCTGA \\
\hline $9[651]$ & AATAGCTGTCACACGCAACGGTACGCCAGCGCTTAATGTAGTA \\
\hline $0[557]$ & TACCTAATATCAAAATCATTCAATATTACGTGA \\
\hline $4[60]$ & TCAGAGGTGTGTCGGCCAGAATGAGTGCACTCTGTGGT \\
\hline $3[476]$ & TTTTTTGTTTAATAAAGTAATTC \\
\hline $8[382]$ & AAGTAAGAGCCGCCAGTACCAGGCGG \\
\hline $3[79]$ & GTGGAACGACGGGCTCTCAACTT \\
\hline $4[102]$ & CCAGCCAAACTTCTGATTGCCGTTTTGGGTAAAGTTAAAC \\
\hline $3[121]$ & AATCAGTTAAAACGTGGGAGAAA \\
\hline $3[224]$ & GCATCAAAAAGAAGTAAATTGGG \\
\hline $7[212]$ & TTTCACGAGAATGACCATTTTCATTTGGTCAATAACCTGT \\
\hline $8[678]$ & CCTACATACGTAGCGGCCAGCCATTGCAACAGGTTTTT \\
\hline $5[539]$ & TTCGCTATTCGCAAGACAAAGTTAATTTCATCTTC \\
\hline $7[17]$ & TTTTTATCCAGCGCAGTGTCACTGC \\
\hline $8[298]$ & CATAGAATTTGCGGTTTGAAAGAGGA \\
\hline $10[79]$ & GTATGTGAAATTGTTATCC \\
\hline
\end{tabular}




\begin{tabular}{|c|c|}
\hline $7[273]$ & ACTACTTAGCCGGAACGAGGCGC \\
\hline $11[387]$ & GGCGACACCACCCTCAGGTTGTACTGTACCGTTCCAGTAA \\
\hline $6[447]$ & TTACCTCTTAGCAAATTTCAACCGATTG \\
\hline $8[508]$ & GGTTTGCGCATTTTAACGCGAGGCGT \\
\hline $10[415]$ & CCTCCGAAATCGGCAAAAT \\
\hline $4[480]$ & TAAGCCAGAGAGCCAGAAGGAAACTCGATAGCCGAACAAA \\
\hline $0[179]$ & GCCTTATACCCTGTAATACCAATTCTTGCGCTC \\
\hline $9[147]$ & CATTCAACCCAAAATGTAGAACCCTCATGAATTAGTACAACC \\
\hline $7[525]$ & TATGTGATAAATAAGGCGTTAAA \\
\hline $0[454]$ & AGACGGGAGAATTGACGGAAATT \\
\hline $11[681]$ & AAAGGGCGCTGGCAAGTATTGGC \\
\hline $4[228]$ & GAGCTTAAGAGGTCCCAATTCTGCAATTCCATATAACAGT \\
\hline $3[331]$ & TACCGGGATAGCAATGAATATAT \\
\hline $4[335]$ & ATTGCGAATAATGTACAACGGAG \\
\hline $2[265]$ & TATGCATTACAGAGGATGGTTTAATTTC \\
\hline $4[564]$ & TTTAGAACGCGAATTACTAGAAAACTATAAACACCGGAAT \\
\hline $11[597]$ & GAGGTAACGTTATTAATTTTAAAACAAATAATGGAAGGGT \\
\hline $5[25]$ & TTTTTCCGGTGCAGCACCGATCCCTTACACTTGCC \\
\hline $1[17]$ & TTTTTTGGTAATGGGTAACCATCCCACTTTTT \\
\hline $8[531]$ & AACGAACCTCCCGACTTGCGGGA \\
\hline $0[515]$ & CTGAAAACCTGTTTATCAAACATGTAACGTCAA \\
\hline $8[592]$ & AAAATTTTTTAAAATGAGCAAAAGAA \\
\hline $7[609]$ & ATTTGGCAAATCAACAGTTGAAA \\
\hline $11[639]$ & CCGATAATAAAAGGGACTTAACACCGCGAACCACCAGCAG \\
\hline $3[583]$ & GGAATCGGAACATTGCACGTTAA \\
\hline $2[349]$ & TGTAGGGGATTTAGTAACACTGAGTTTC \\
\hline $3[434]$ & AAAAGGGCGACAATTATTTATCC \\
\hline $5[371]$ & ATCAGAGCCTTTAACGGGGTCTTAATGCCCCCTGC \\
\hline $7[338]$ & GGAGCAGCCACCACCCTTCGCATAACGACAATGACAACAA \\
\hline $3[56]$ & ATCAGCGGGGTCAGCTTTCAGAG \\
\hline $0[473]$ & AAAAAAGGCAGCCTTTACAATCTTACCAGTTTG \\
\hline $6[698]$ & TTTTTAACAATATTACCGTCGCTGGTAATATCCAGTTTTT \\
\hline $8[88]$ & AGCCTCCCCAGGGTCCGGCAAACGCG \\
\hline $6[405]$ & CAAGTGCTGAGTAAGAAAATAAATCCTC \\
\hline $7[632]$ & GGAATAACAGAGATAGACATACAAACTTGAGGATTTAGAA \\
\hline $0[76]$ & GACTTTCTCCGTGGCGCGGTTG \\
\hline 2[97] & GCGAAAGACGCAAAGCCGCCACGGGAAC \\
\hline $4[648]$ & GCATCGAGCCAGATATCTTTAGGACCTGAGGAAGGTTATC \\
\hline $4[606]$ & ACAGTTTTTCAGATTTCAATTACCGTCGCAGAGGCGAATT \\
\hline $7[548]$ & TAAGATCTGTAAATCGTTGTTAATTGTAAAGCCAACGCTC \\
\hline $11[555]$ & CCCACATGTGAGTGAATAACTGATGCTTTTAACCTCCGGC \\
\hline $9[399]$ & ATAAGAAGCCACCCAAACTTGAGCCATTATCAATACATCAGT \\
\hline $11[189]$ & ACTGCCCGCTTTCCTGAAAAGCTATATTTTAAATA \\
\hline $3[499]$ & TGTCCAAGTACCAGAAACCCCAG \\
\hline $4[209]$ & AATGCTGTAGCTGAGAAAGGCCG \\
\hline $7[357]$ & GTGTATTAAGAGGCTGAGACTCC \\
\hline
\end{tabular}




\begin{tabular}{|c|c|}
\hline $8[237]$ & GCTTGACCATTAGATACATTTCG \\
\hline $9[609]$ & GATGAATAAATCCTGTAGGTGAGGCGGTAGCGTAAGTCCTCA \\
\hline $0[328]$ & TTGTCGTCTTTCTACGTAATGCC \\
\hline $11[513]$ & CTCCAATTTAGGCAGAGACAATCAATCAAGAAAAATAATA \\
\hline $3[560]$ & AAGACGCTGAGACCAGAAGGAGC \\
\hline $7[42]$ & GCGCCTGAATGCCAACGGCCCAGCCTCCCGCGTGCCTGTTCTTCTTTTT \\
\hline $0[370]$ & GCGTCATACATGCCCTCATAGTT \\
\hline $11[303]$ & GTGAGTTAAAGGCCGCTGACACTCATGAAGGCACCAACCT \\
\hline $3[373]$ & GGTCACGCCAGCACAGGAGTTAG \\
\hline $4[51]$ & GGGTTACCTGCAGCCAGCGGTGTTTTT \\
\hline $7[254]$ & TTACCAATAAGGCTTGCAGTGCGGAAGTTTAGACTGGATA \\
\hline $8[466]$ & GGCATAAGCGTCTTCGAGGAAACGCA \\
\hline $3[247]$ & CTTGAAAACACCCTAACGGCATA \\
\hline $8[405]$ & GGTGCCGTCGAGAGGGTTGATAT \\
\hline $10[331]$ & TCGTTCACCGCCTGGCCCT \\
\hline $8[615]$ & GTTGAAACAAACATCAAGAAAAC \\
\hline $6[531]$ & GACCGTCGAACGGGGAAGCTAATGCAGA \\
\hline $6[363]$ & TGAAATTGTTTCAGGGAACTACAACGCC \\
\hline $10[625]$ & AACACCCTAAAGGGAGCCC \\
\hline $6[279]$ & CATGTCAGAGATTTGATGTGAATTACCT \\
\hline $11[429]$ & CCCTTCATATAAAAGAACGTAGAGCCTTAAAGGTGAATTA \\
\hline $11[651]$ & TTGACGGGGAAAGCTTCACCAGAAATGGCATCACT \\
\hline $6[615]$ & GTCAGTCGTTTAACGAGATGGCAATTCA \\
\hline $7[422]$ & AGCGCCACCACGGAATACGCCTCAGACCAGAGCCACCACC \\
\hline $4[312]$ & ATTTGCCAAGCGGAACTGACCAACGAGTCAATCATAAGGG \\
\hline $8[550]$ & CAGTAAGAACCTTGAGCCTGTTTAGT \\
\hline $4[503]$ & AGCAAGCCGTTTAAGAATTGAGT \\
\hline $2[601]$ & TCAATAATAAAGTGTATCATCATATTCC \\
\hline $9[21]$ & TTTTTGCGTCCGTGCCTGCATCAGACGTTTTT \\
\hline $11[483]$ & GAACAAGAGTCCACCAATTTTTTAGTTGTCGTAGG \\
\hline $10[499]$ & CTATATTAAAGAACGTGGA \\
\hline $4[186]$ & GAGACAAAGATTATCAGGTCATTGACGAGAGATCTACAAA \\
\hline $9[63]$ & TTCACCTAGCGTGGCGGGTGAAGGGATACCAGTGCATAAAAA \\
\hline $11[609]$ & AGCACTAAATCGGATCGTATTTAGACTTATATCTG \\
\hline $4[293]$ & AAATTGTGTCGAGAATACCACAT \\
\hline $3[667]$ & GGCGCCCCGCCGAATCCTGAGAAGTGAGGCCGATTAAAGG \\
\hline $3[205]$ & GTCAGAATCAGGCAGGATTCGCG \\
\hline $0[622]$ & AAGATAAAACAGTTGGATTATAC \\
\hline $6[111]$ & TCAGGTGAAATTTCTACGGAAACAATCG \\
\hline $10[205]$ & AGCAGTCGGGAAACCTGTC \\
\hline $6[489]$ & AATCATAATAACCCGGCGTCAAAAATGA \\
\hline $0[496]$ & TCCCATCCTAATGAGAATAACAT \\
\hline $0[221]$ & CGAGCACAGACTTCAAATACCTCAAAAGCTGCA \\
\hline $9[231]$ & TTAGTGTGAATCCCTCTAATAAAACGAAAGAACGATGAATTA \\
\hline $4[629]$ & CAAATATCAAACCAGATGAATAT \\
\hline $0[664]$ & GATTTTAGACAGGCATTAAAAATA \\
\hline
\end{tabular}




\begin{tabular}{|c|c|}
\hline $10[667]$ & AGACGGCGAACGTGGCGAG \\
\hline $0[599]$ & TTCTGGAATAATCCTGATTTTGCCCGGCCGTAA \\
\hline $3[23]$ & TTTGCAACCAGCTTACGGCGGTGGTGAGGTTTCAGTTGAGGATCCTTTTT \\
\hline $8[340]$ & GCGCCCGCACCCTCTCGAGGTGAATT \\
\hline $7[674]$ & GCCTTACGCTGCGCGTAAAATTATTTTTTGACGCTCAATC \\
\hline $7[86]$ & ATGAATCCCAGTCACGATCGAACGTGCCGGCCAGAGCACA \\
\hline $5[245]$ & CGCCTGACGGTAGAAAGATTCTAATGCAGATACAT \\
\hline $8[657]$ & GTATTAGAGCCGTCAATAGATAA \\
\hline $3[308]$ & CTAAAGACTTTTAGGAACCCATG \\
\hline $2[702]$ & TTTTTTATAACGTGCTTTCCTCTTTATAACAGTACTAT \\
\hline $4[671]$ & TACTTCTTTGATAAAAATCTAAA \\
\hline $2[391]$ & ATTAAAATAAGTGCGACGATTGGCCTTG \\
\hline $9[189]$ & GAGCAAGGTGGCATTTACTCCAACAGGTTCTTTACGTCAACA \\
\hline $4[167]$ & CAATATGATATTGATGGGCGCAT \\
\hline $7[147]$ & GCTAATGCCGGAGAGGGTAGCTA \\
\hline $7[464]$ & AAGCACAGAGCCTAATTATTGTTAGCGATTAAGACTCCTT \\
\hline $8[172]$ & TAATCGTAGCATTACCTGAGAGTCTG \\
\hline $0[580]$ & TAGAACCTACCAGTCTGAGAGAC \\
\hline $4[354]$ & GAAAGTTCAACAATCAGCTTGCTTAGCTTTAATTGTATCG \\
\hline $8[46]$ & CAGCATCAACCGCACGGCGGGCCGTT \\
\hline $2[181]$ & TTATGGCCTGAGCACCTCAGAGCATAAA \\
\hline $3[644]$ & CTATTAGTCTTTCGCCGCTACAG \\
\hline $8[111]$ & CTTTTTTTCGTCTCGTCGCTGGC \\
\hline $11[231]$ & TTAATGAATCGGCCATTCATTCCAATACGCATAGT \\
\hline $3[518]$ & AACAACATGTTCATCCTTGAAAA \\
\hline $5[77]$ & AACGTTGTAGAAACAGCGGATAGTTGGGCGGTTGT \\
\hline $10[706]$ & TTTTTAGGAGCGGGCGCTAGGAAGGGAAGAAAGCGAATTTTT \\
\hline $9[441]$ & TGCCATACATAAAGATTAACTGAACACCAACAGCCGGAATAG \\
\hline $7[189]$ & GGCTAAAGTACGGTGTCTGGAAG \\
\hline $6[237]$ & AAGAGATTCATTTTGTTTAAGAGGAAGC \\
\hline $5[203]$ & TGTAAATCATGCTCCTTTTGATAATTGCTGAATAT \\
\hline $7[315]$ & AATCCAAAAAAAAGGCTCCAAAA \\
\hline $10[583]$ & TGGCAAGTTTTTTGGGGTC \\
\hline $2[559]$ & GAATTATCCAATAACGATAGCTTAGATT \\
\hline $11[364]$ & GTCCACGCGCCACCTCACCGTTGAAACA \\
\hline $11[471]$ & TGTTCCAACGCTAACGAACAAGTCAGCAGGGAAGCGCATT \\
\hline $4[522]$ & ACCGCATTCCAACGGTATTCTAAGCGAGATATAGAAGGCT \\
\hline $7[380]$ & TCAAGCAGAACCACCACTCACTCAGGTAGCCCGGAATAGG \\
\hline $8[447]$ & ATTCTTTTCATAATCAAAATCAC \\
\hline $6[321]$ & AAATCCCCGAAACAATTCATGAGGAAGT \\
\hline $10[541]$ & CATTCTATCAGGGCGATGG \\
\hline $10[373]$ & TACCTGGTTTGCCCCAGCA \\
\hline $5[413]$ & AGAGTTTATACCAGTAGCACCTGAAACCATCGATA \\
\hline $9[105]$ & GTCCGTCCTGCAAGATCGTCGGATTCTCTTCGCATTGGACGA \\
\hline $11[63]$ & ATAGCTGTTTCCTGGAACGTCCATAACGCCGTAAA \\
\hline $11[177]$ & TGCGTACTAATAGTAGTTGAAATGCATATTTCAACGCAAG \\
\hline
\end{tabular}




\begin{tabular}{|c|c|c|}
\hline $8[702]$ & TTTTTAAAAACGCTCATGGAAATA & \\
\hline $7[441]$ & TTGAAGCCCTTTTTAAGAAAAGT & \\
\hline $11[525]$ & AGGGCGAAAAACCGATTTAACGTAGGGCAAATACC & \\
\hline $2[475]$ & AAATAGGTAATTTACAAATAAGAAACGA & \\
\hline $9[525]$ & TTTAGCAAACGCCACAATATAACTATATTCCCTTATAAATGG & \\
\hline $7[399]$ & TATTGCCTTTAGCGTCAGACTGT & \\
\hline $0[389]$ & GAATTGTAGCCAGAATGGATCAGAGCAAATCCT & \\
\hline $2[307]$ & TTCCATTGACCCAAAGAGGCTTTGAGGA & \\
\hline $7[651]$ & TAAGTAGAAGAACTCAAACTATCG & \\
\hline $2[517]$ & ACGCGTCGGCTGTAAGACGACGACAATA & \\
\hline $7[483]$ & GTTTACCGCGCCCAATAGCAAGC & \\
\hline $2[55]$ & TTCGCCATAAACTCTGGAGGTGTCCAGC & \\
\hline $10[48]$ & GCAGCACTTTGCTCTGAGCCGGGTCACTGTTGCCCTGCGGCTTTTT & \\
\hline $6[657]$ & TGCCTGAACAGCAAATGAATGCGCGAACT & \\
\hline $3[163]$ & TAAAGAGGCAAAATATTTTATAA & \\
\hline $0[538]$ & TTAGGTTGGGTTATAGATAAGTC & \\
\hline $4[419]$ & GCAGCACCGTAAGTGCCCGTATA & \\
\hline $8[214]$ & CAAATGGTTCAGAAGAACGAGTAGAT & \\
\hline $3[415]$ & GTTTATGTCACATGGGAATCCAC & \\
\hline $0[641]$ & CCGAACCCCCTAAAACATCGACCAGTTTAGAGC & \\
\hline $8[321]$ & CCGAACGGTGTACAGACCAGGCG & \\
\hline $3[457]$ & CAATCCAAAATACTGAACAGTAG & \\
\hline $6[195]$ & TGCAACTCAAAAGGCCGTACCAAAAACA & \\
\hline $0[95]$ & CCGGAAGACGTACAGCGCCGCGATTACAATTCC & \\
\hline $11[399]$ & GTTTGATGGTGGTTCAGAACCCCGCCTCACAGAAT & \\
\hline $11[25]$ & TTTTTCCGGGTACCGAGCTCGAATTCGTAATCTGGTCA & \\
\hline $0[53]$ & CGGTAGTACTCAATCCGCTGCTGGTCATGGTC & \\
\hline $8[256]$ & AAAATTCCATTCAGGCTTTTGCAAAAGAAGTCA & \\
\hline $3[266]$ & AACTTTAATCATGGGTAGCAACGGCTACGACAGCAACTAAAA & \\
\hline $10[247]$ & AATAACGCGCGGGGAGAGGCGGTT & \\
\hline $0[251]$ & TGGGAAGAAAAATCTACGTGCGTTTTAATT & \\
\hline $0[263]$ & CAGTCTTGATTTTAAGAAC & \\
\hline $8[286]$ & GACCTTCATTTTGCCAGAGGGGGTAATAGT & \\
\hline $7[296]$ & AGACGTCGTCACCCTCAGACCTGCTC & \\
\hline $10[293]$ & ACCGGATGTTTTTCTTTTCACCA & $\begin{array}{l}\text { ATTO } 647 \mathrm{~N} \text {, only used } \\
\text { for independent } \\
\text { blinking }\end{array}$ \\
\hline $10[286]$ & ATTCATTAGAGTAATCTTGACGCTGGCT & \\
\hline $4[461]$ & AAGAAACAATGACCGGAAACGTC & biotin labeled \\
\hline $4[83]$ & GTACATCGACATCGTTAACGGCA & biotin labeled \\
\hline $5[665]$ & ATACCACCATCAGTGAGGCCAAACCGTTGTAGCAA & biotin labeled \\
\hline $4[251]$ & AACGCCAAAAGGCGGATGGCTTA & biotin labeled \\
\hline $5[119]$ & CATAATATTCCGTAATGGGATCCGTGCATCTGCCA & external labeling \\
\hline $3[98]$ & GGATAACCTCACAATTTTTGTTA & external labeling \\
\hline $4[125]$ & GTTTGAGGGGACCTCATTTGCCG & external labeling \\
\hline $4[144]$ & CGTAAAGGTCACGAAACCAGGCAATAGCACCGCTTCTGGT & external labeling \\
\hline
\end{tabular}




\begin{tabular}{|l|l|l|}
\hline $0[137]$ & CATCAGCGTCTGGCCTTCCACAGGAACCTGGGG & external labeling \\
\hline $10[121]$ & GGGCCGGAAGCATAAAGTG & external labeling \\
\hline $11[135]$ & TAAAGGATTGTATAAGCGCACAAACGACATTAAATGTGAG & external labeling \\
\hline $7[128]$ & TTCCGAATTGTAAACGTGTCGCCAGCATCGGTGCGGGCCT & external labeling \\
\hline $3[140]$ & CAATAGGAACGCAAATTAAGCAA & external labeling \\
\hline $7[105]$ & GAAAGATCGCACTCCAGCCAGCT & external labeling \\
\hline $0[160]$ & GATAAAAATTTTAGCCAGCTTT & external labeling \\
\hline $8[153]$ & TCAGGCTGCGCAACTGTTGGGAA & external labeling \\
\hline $0[118]$ & CGAGTAACAACCGTTTACCAGTC & external labeling \\
\hline $2[139]$ & TTCGCGGATTGATTGCTCATTTTTTAAC & external labeling \\
\hline $10[279]$ & ACCCAAATGGCAAAAGAATACTCGGAACAGAATCC & $\begin{array}{l}5 \text { ' ATTO } 647 N, \text { only } \\
\text { used for collective } \\
\text { blinking }\end{array}$ \\
\hline $10[265]$ & AACAAAGCTGCTGTAACAACAAGGACGT & $\begin{array}{l}5^{\prime} \text { ATTO 647N } \\
5 ' \text { ATTO 700, only } \\
\text { used for collective } \\
\text { blinking }\end{array}$ \\
\hline $10[272]$ & TCAACGTTGCGTATTGGGCGCCAGGGTG & \\
\hline
\end{tabular}

\section{REFERENCES}

1. Nickels, P. C.; Wünsch, B.; Holzmeister, P.; Bae, W.; Kneer, L. M.; Grohmann, D.; Tinnefeld, P.; Liedl, T. Molecular Force Spectroscopy with a DNA Origami-Based Nanoscopic Force Clamp. Science 2016, 354, 305-307.

2. Derr, N. D.; Goodman, B. S.; Jungmann, R.; Leschziner, A. E.; Shih, W. M.; Reck-Peterson, S. L. Tug-of-War in Motor Protein Ensembles Revealed with a Programmable DNA Origami Scaffold. Science 2012, 338, 662-665.

3. Laurence, T. A.; Fore, S.; Huser, T. Fast, Flexible Algorithm for Calculating Photon Correlations. Opt. Lett. 2006, 31, 829-831.

4. Schröder, T.; Scheible, M. B.; Steiner, F.; Vogelsang, J.; Tinnefeld, P. Interchromophoric Interactions Determine the Maximum Brightness Density in DNA Origami Structures. Nano Lett. 2019, 19, 1275-1281.

5. Zumbusch; Fleury; Brown; Bernard; Orrit. Probing Individual Two-Level Systems in a Polymer by Correlation of Single Molecule Fluorescence. Phys. Rev. Lett. 1993, 70, 3584-3587.

6. Orrit, M. Photon Statistics in Single Molecule Experiments. Single Mol. 2002, 3, 255-265.

7. Lamb, D. C.; Schenk, A.; Röcker, C.; Nienhaus, G. U. Determining Chemical Rate Coefficients Using Time-Gated Fluorescence Correlation Spectroscopy. J. Phys. Org. Chem. 2000, 13, 654-658.

8. Yu, J.; Lammi, R.; Gesquiere, A. J.; Barbara, P. F. Singlet-Triplet and Triplet-Triplet Interactions in Conjugated Polymer Single Molecules. J. Phys. Chem. B 2005, 109, 10025-10034. 9. Hedley, G. J.; Schröder, T.; Steiner, F.; Eder, T.; Hofmann, F. J.; Bange, S.; Laux, D.; Höger, S.; Tinnefeld, P.; Lupton, J. M.; Vogelsang, J. Picosecond Time-Resolved Photon Antibunching Measures Nanoscale Exciton Motion and the True Number of Chromophores. Nat. Commun. 2021, 12, 1327. 